http://dx.doi.org/10.32929/2446-8355.2019v28n4p505-515

\title{
BIOATIVIDADE DE EXTRATOS AQUOSOS DE Hedychium coronarium J. KOENIG SOBRE Spodoptera frugiperda J. E. SMITH, 1797 (Lepidoptera: Noctuidae)
}

\section{Gláucia Maria Pereira Pavarini ${ }^{1 *}$, Izabella Victoriano de Souza ${ }^{2}$, Ronaldo Pavarini $^{1}$, João Vicente Coffani-Nunes ${ }^{1}$}

\footnotetext{
${ }^{1}$ Docente do curso de Engenharia Agronômica, Universidade Estadual Paulista, Campus Experimental de Registro, Registro, SP. *E-mail do autor correspondente: glaucia.pavarini@unesp.br

${ }^{2}$ Discente do curso de Engenharia Agronômica, Universidade Estadual Paulista, Campus Experimental de Registro, Registro, SP.
}

Recebido: 21/02/2019; Aceito: 16/12/2019

RESUMO: O estudo teve como objetivo avaliar a atividade inseticida dos extratos aquosos da raiz, rizoma, bainha e lâmina foliar da planta Hedychium coronarium J. Koenig, para lagartas do $3^{\circ}$ instar de Spodoptera frugiperda J. E. Smith, 1797, considerada principal praga da cultura do milho no Brasil. Os tratamentos consistiram de extratos aquosos das partes vegetais já mencionadas, preparados na concentração de $10 \%(\mathrm{p} / \mathrm{v})$. Como tratamento controle foi utilizado água destilada. Cubos de dieta artificial de $1 \mathrm{~cm}^{3}$ foram mergulhadas nos extratos aquosos e na água por dez segundos sendo em seguida ofertados as lagartas. As características avaliadas foram massa, sobrevivência e duração da fase larval e pupal, massa do inseto adulto e ciclo biológico ovo-adulto. Os extratos aquosos obtidos das partes vegetais de $H$. coronarium não apresentaram toxicidade à $S$. frugiperda. Ao contrário do que se esperava, houve estímulo da alimentação quando as lagartas foram submetidas ao extrato aquoso de folhas desta espécie vegetal, apresentando maior massa larval e antecipação do ciclo biológico.

Palavras-chave: Planta inseticida. Lagarta-do-cartucho. Inseticida botânico.

\section{BIOACTIVITY OF AQUEOUS EXTRACTS OF Hedychium coronarium J. KOENIG ON Spodoptera frugiperda J. E. SMITH, 1797 (Lepidoptera: Noctuidae)}

\begin{abstract}
The aim of this study was to evaluate the insecticidal activity of aqueous extracts of root, rhizome, sheath and leaf of the plant Hedychium coronarium J. Koenig for 3rd instar's Spodoptera frugiperda caterpillars J.E. Smith, 1797, which is considered the main pest of corn in Brazil. The treatments consisted of aqueous extracts of the aforementioned plant parts, prepared in the concentration of $10 \%(\mathrm{w} / \mathrm{v})$. As a control treatment, it was used distilled water. Cubes of $1 \mathrm{~cm}^{3}$ artificial diets were immersed in aqueous extracts and water for ten seconds and then caterpillars were offered. The characteristics evaluated were larval end pupal mass, survival and duration, adult insect mass end egg-adult biological cycle. The aqueous extracts obtained from the $H$. coronarium plant parts showed no toxicity to $S$. frugiperda. Contrary to what was expected, feeding was stimulated when the caterpillars were submitted to aqueous extract of leaves of this plant species, presenting larger larval mass and anticipation of the biological cycle.
\end{abstract}


Key words: Plant insecticide. Armyworms. Botanical insecticide.

\section{INTRODUÇÃO}

Um dos principais fatores que comprometem o rendimento e a qualidade da produção da cultura do milho é a incidência de insetos pragas. Dentre estes, destacam-se os insetos imaturos da ordem Lepidoptera, ou seja, as lagartas (BARROS, 2012), como a lagarta-docartucho, Spodoptera frugiperda, a lagarta-da-espiga, Helicoverpa zea e a broca-da-cana-deaçúcar, Diatraea saccharalis (SÂMIA et al. 2016; MICHELOTTO et al., 2013).

A lagarta-do-cartucho é considerada a principal praga da cultura do milho (LIMA JUNIOR et al., 2012), ocorrendo em muitas regiões produtoras, como nos Estados Unidos (FARIAS, 2010), e no Brasil (MARTINELLI et al., 2006; NAGOSHI et al., 2007), o inseto está sempre frequente desde a emergência da plântula até a formação das espigas. Os prejuízos causados pela lagarta-do-cartucho dependem do estágio em que a planta se encontra no momento do ataque. Assim, até os 30 dias de desenvolvimento da cultura podem ser esperados prejuízos da ordem de $15 \%$, já no florescimento podem chegar a 34\% (BARROS, 2012).

A elevada densidade populacional causa destruição das folhas e principalmente do cartucho das plantas, comprometendo assim a produtividade e rendimento de grãos (PENCOE; MARTIN, 1981). Estima-se que as perdas de produção causadas pela lagarta na cultura variam de 34 e 52\% (CRUZ et al., 1997).

Para realização do controle de Spodoptera frugiperda é frequente o uso de inseticidas organosintéticos, entretanto este método tem apresentado eficiência reduzida. Isto se deve a diversos fatores dentre eles o surgimento de populações resistentes, em consequência da pulverização subsequente de produtos com o mesmo mecanismo de ação. Em algumas regiões além da elevada pressão de seleção por inseticidas há o aparecimento de problemas vinculados com as proteínas de Bacillus thuringiensis, propiciando um cenário favorável para a evolução da resistência, o que compromete com esta estratégia de controle (FARIAS, 2010).

Os problemas causados pelos inseticidas sintéticos induziram as pesquisas com plantas inseticidas pela necessidade de se encontrar substâncias químicas que apresentem eficiência de controle das pragas (BOGORNI; VENDRAMIM, 2003). A diminuição na diversidade de moléculas sintéticas com atividade inseticida e os incrementos nos custos de produção reforçam o interesse na busca de alternativas de biocidas de origem vegetal (VENDRAMIM; CASTIGLIONI, 2000). A implantação do manejo integrado de pragas, bem como o crescimento da agricultura orgânica também criaram um ambiente propício para o desenvolvimento de trabalhos com plantas com ação inseticida, as quais são alternativas no controle de pragas.

Há diversas substâncias químicas provenientes do metabolismo secundário de espécies vegetais que desempenham papel defensivo, podendo causar inibição da ação de insetos herbívoros, seja por inibir a alimentação, redução da oviposição e prejudicar o crescimento na fase larval (BOEKE et al., 2004; KETOH et al., 2005; ISMAN, 2006). Os inseticidas de origem vegetal utilizados em forma de pó, óleo e extrato, são considerados promissores 
podendo ter efeitos por contato, ingestão, fumigação (ALMEIDA et al., 2005; SOUSA et al., 2005; BRITO et al., 2006;).

Zingiberácea é uma família de monocotiledôneas que compreende plantas herbáceas, com rizoma e folhas lanceoladas (BALTAZAR, 1994), tendo como exemplar mais conhecido popularmente o gengibre. Segundo Rohde et al., (2013) o extrato aquoso de gengibre apresentou propriedades inseticidas sobre larvas e pupas de Ceratitis capitata. Outra espécie pertencente a esta família, conhecida como lírio-do-brejo (Hedychium coronarium) apresentou propriedades repelentes ao gorgulho-do-milho Sitophylus zeamais (AGUIAR et al., 2015).

Considerando a importância de S. frugiperda na cultura do milho, bem como, o potencial inseticida de plantas da família Zingiberaceae, este trabalho objetivou avaliar o efeito de extratos aquosos de partes vegetais da espécie Hedychium coronarium, conhecida popularmente como lírio-do-brejo, sobre características biológicas desse inseto.

\section{MATERIAL E MÉTODOS}

\section{Coleta, identificação e processamento do material vegetal}

Plantas de lírio-do-brejo (Hedychium coronarium) foram coletadas no município de Registro, SP, em local georeferenciado por meio de GPS (Sistema de Posicionamento Global): latitude Sul $24^{\circ} 32^{\prime}$ e longitude oeste $47^{\circ} 51^{\prime}$, no dia 20 de abril de 2016 . Após a coleta as plantas foram encaminhadas ao laboratório de Fitossanidade da UNESP, Campus de Registro, onde o material foi lavado em água corrente retirando todas as impurezas e na sequencia realizou-se a separação das partes vegetais: raiz, rizoma, bainha e lâmina foliar.

Em seguida o material foi armazenado individualmente em sacos de papel, previamente identificados e depositados em estufa de ventilação forçada a $40^{\circ} \mathrm{C}$ onde foram mantidos até que apresentassem massa constante. Após a secagem o material foi submetido a um triturador de facas para obtenção do pó vegetal de cada parte da planta. Estes materiais foram acondicionados individualmente em recipientes de vidro etiquetados, hermeticamente fechados e envoltos com papel alumínio até serem utilizados nos bioensaios.

Parte do material vegetal coletado foi utilizado para confecção de uma exsicata, obtendo-se o material testemunho (voucher) que foi utilizada para a confirmação da espécie pelo Prof. Dr. João Vicente Coffani Nunes. Esta exsicata foi depositada no Herbário SPVR na UNESP - Câmpus de Registro, identificada com número SPVR1731.

\section{Preparação dos extratos aquosos}

Para obtenção do extrato aquoso foram pesados individualmente 10 gramas de pó de cada parte vegetal a ser testada, sendo transferidos individualmente para erlenmeyer de vidro de $100 \mathrm{~mL}$, completando-se o volume com água destilada, e obtendo-se um preparado com $10 \%$ (massa/volume). A mistura foi agitada manualmente por um minuto, visando boa homogeneização dos componentes. Em seguida o recipiente foi fechado com filme plástico e envolto com papel alumínio sendo armazenado em refrigerador a $4{ }^{\circ} \mathrm{C}$ por período de $24 \mathrm{~h}$. Após este período o material obtido foi filtrado em tecido de malha fina, obtendo-se assim o 
líquido denominado de extrato aquoso. Este líquido foi utilizado no ensaio biológico por um período não superior a 24 horas após a sua obtenção.

\section{Criação de Spodptera frugiperda em laboratório}

A criação de $S$. frugiperda foi mantida em laboratório em câmara climatizada sob temperatura de $25 \pm 2^{\circ} \mathrm{C}$; UR de $70 \pm 10 \%$ e fotofase de 14 horas, conforme descrito por Sâmia et al. (2016).

\section{Efeito do extrato aquoso de $H$. coronarium sobre parâmetros biológicos de $S$. frugiperda}

$\mathrm{O}$ experimento foi desenvolvido em ambiente climatizado à temperatura de $25 \pm 2{ }^{\circ} \mathrm{C}$, umidade relativa de $70 \pm 10 \%$ e fotofase de 14 horas. O delineamento experimental utilizado foi inteiramente casualizado composto por 5 tratamentos (Tabela 1) e 5 repetições, cada uma composta por cinco lagartas de terceiro instar de S. frugiperda.

Tabela 1. Tratamentos testados no bioensaio envolvendo extrato aquoso das estruturas vegetativas de Hedychium coronariumem Spodoptera frugiperda. Treatments tested in the bioassay involving aqueous extract of the vegetative structures of Hedychium coronarium in Spodoptera frugiperda.

\begin{tabular}{ll}
\hline Tratamento & Descrição \\
\hline Controle & Dieta artificial + água destilada \\
ER $^{(1)}$ & Dieta artificial + extrato aquoso da raiz de H. coronarium \\
ERZ & Dieta artificial + extrato aquoso do rizoma de H. coronarium \\
EB & Dieta artificial + extrato aquoso da bainha foliar de H. coronarium \\
EF & Dieta artificial + extrato aquoso da lâmina foliar de H. coronarium \\
\hline
\end{tabular}

${ }^{(1)}$ ER: extrato de raiz; ERZ, extrato de rizoma; EB, extrato de bainha; EF, extrato de lâmina foliar. ${ }^{(l)}$ ER: root extract; ERZ, rhizome extract; EB, sheath extract; EF, leaf blade extract.

Fonte: Autoria própria. Own authorship.

A dieta que foi utilizada no bioensaio foi preparada de acordo com Greene et al. (1976) e, após o resfriamento, foi moldada em cubos de $1 \mathrm{~cm}^{3}$. Estes cubos de dieta artificial foram mergulhados por 10 segundos nos recipientes com os diferentes extratos aquosos (tratamentos), sendo em seguida colocados sobre uma folha de papel toalha visando eliminar o excesso de umidade. Por meio de ensaio preliminar, mergulhando cubos de dieta (sólida) nos extratos aquosos testados (para as diferentes partes vegetais na mesma concentração), observou-se que ocorreu aumento do peso dos cubos, indicando que houve absorção do extrato aquoso e que estes extratos estavam incorporados a dieta artificial e disponíveis para serem ingeridos pelas lagartas, principalmente na periferia do cubo. Considerando que o comportamento alimentar da lagarta é de ingerir, preferencialmente, a dieta presente na periferia do cubo, pode-se afirmar que houve a ingestão dos extratos testados.

$\mathrm{Na}$ sequência os cubos de dieta tratados com os diferentes extratos foram acondicionados individualmente em recipientes de plástico transparente de $100 \mathrm{~mL}$, previamente identificados. Em seguida em cada recipiente foi liberada uma lagarta de terceiro instar de $S$, frugiperda. Estes recipientes foram armazenados em câmera climatizada sob temperatura de $25 \pm 2^{\circ} \mathrm{C}$; UR de $70 \pm 10 \%$ e fotofase de 14 horas. 
O peso das lagartas foi determinado com auxílio de balança analítica, aos 12 dias após a exposição (DAE) das lagartas aos tratamentos. O peso da fase pré-pupal também foi determinado. Quando as lagartas atingiram a fase pupal foi determinada a duração e viabilidade larval.

Após 24 horas da pupação as pupas foram pesadas e acondicionadas individualmente em recipientes plásticos de $100 \mathrm{~mL}$ e observadas diariamente até a emergência dos adultos, para a determinação da duração e viabilidade da fase pupal. No dia seguinte da emergência do adulto o mesmo foi sacrificado por exposição a baixa temperatura em freezer, possibilitando assim sua pesagem em balança analítica.

\section{Análises dos dados}

Os dados relativos massa larval, duração dos estágios larval e pupal, viabilidade larval, massa e viabilidade de pupas, massa dos adultos e ciclo biológico total foram submetidos à análise de variância (ANOVA), e as médias foram comparadas aplicando-se teste Tukey ao nível de significância de 5\%, utilizando o software SISVAR (FERREIRA, 2011).

\section{RESULTADOS E DISCUSSÃO}

O tratamento constituído por dieta artificial incorporado com extrato aquoso das folhas de Hedychium coronarium induziu nas lagartas um aumento de massa corporal se comparado com o tratamento testemunha constituído de dieta artificial incorporado água destilada. $\mathrm{O}$ peso das lagartas aos 12 dias após a exposição aos diferentes extratos aquosos variou de 351,0 a 462,0 mg, respectivamente para a dieta submersa em água destilada e dieta submersa em extratos aquosos das folhas. É provável que alguma substância presente no extrato aquoso e ingerida pela lagarta tenha exercido um efeito fago estimulante, provocando acréscimo da massa corporal no inseto imaturo. $\mathrm{Na}$ fase de pré-pupa e pupa não houve diferença significativa entre os tratamentos testados quanto à massa, respectivamente (Tabela 2).

Os pesquisadores Tanzubil e McCafferry (1990), descrevem que a massa da lagarta e a duração da fase larval estão relacionadas com o potencial fago inibidor do bioinseticida ou no seu envolvimento com o metabolismo hormonal da lagarta, fazendo com que o inseto tenha dificuldade de passar de um instar para o outro. Em estudo realizado por Mordue e Nisbet (2000), a Azadirachtina, uma molécula presente nos extratos de Nim, atuou inibindo a alimentação e interferindo no crescimento de várias espécies de insetos.

Apesar de os extratos aquosos de $H$. coronarium terem provocado alteração na massa corporal das lagartas, os mesmos não afetaram a sobrevivência desse inseto na fase larval, nem tampouco provocaram alteração na duração do estágio larval (Tabela 3). 
Tabela 2. Médias $( \pm \mathrm{EP})^{1}$ de massa da fase larval, pré-pupal e pupal de Spodoptera frugiperda criada em dieta artificial tratadas com extratos aquosos a $10 \%$ de diferentes estruturas vegetativas de Hedychium coronarium. Mean $( \pm S E)^{l}$ of larval, pre-pupal and pupal phases of Spodoptera frugiperda grown in an artificial diet treated with aqueous extracts at $10 \%$ of different vegetative structures of Hedychium coronarium.

\begin{tabular}{lccc}
\hline \multirow{2}{*}{ ESTRUTURAS } & \multicolumn{3}{c}{ Massa (miligramas) } \\
\cline { 2 - 4 } & Larval 12 DAE & Pré-Pupa & Pupa \\
\hline Testemunha & $351,0 \pm 13,27 \mathrm{~b}$ & $329,2 \pm 5,72 \mathrm{a}$ & $218,8 \pm 3,62 \mathrm{a}$ \\
Raiz & $410,0 \pm 20,02 \mathrm{ab}$ & $266,0 \pm 13,98 \mathrm{a}$ & $228,2 \pm 9,09 \mathrm{a}$ \\
Rizoma & $437,0 \pm 21,05 \mathrm{ab}$ & $272,6 \pm 2,25 \mathrm{a}$ & $248,6 \pm 5,64 \mathrm{a}$ \\
Bainha & $457,0 \pm 33,97 \mathrm{ab}$ & $254,2 \pm 6,52 \mathrm{a}$ & $234,8 \pm 6,48 \mathrm{a}$ \\
Lâmina Foliar & $462,0 \pm 33,80 \mathrm{a}$ & $310,0 \pm 5,72 \mathrm{a}$ & $247,8 \pm 6,67 \mathrm{a}$ \\
\hline Valor de $p$ & $0,0381^{*}$ & $0,0631^{\mathrm{NS}}$ & $0,0629^{\mathrm{NS}}$ \\
\hline \multicolumn{1}{c}{$\mathrm{CV}(\%)$} & 13,59 & 15,21 & 7,45 \\
\hline
\end{tabular}

*Médias seguidas pela mesma letra na coluna não diferem entre si pelo teste de Tukey $(\mathrm{P} \leq 0,05)$; NS = não significativo. ${ }^{1} \mathrm{EP}=$ Erro Padrão. ${ }^{*}$ Means followed by the same letter in the column do not differ by Tukey test $(P$ $\leq 0.05) ; N S=$ not significant. ${ }^{l} S E=$ standard error.

Fonte: Autoria própria. Own authorship.

Tabela 3. Médias $( \pm \mathrm{EP})^{1}$ de duração e viabilidade da fase larval de Spodoptera frugiperda criada em dieta artificial tratadas com extratos aquosos a $10 \%$ de diferentes estruturas vegetativas de Hedychium coronarium. Averages $( \pm S E)^{1}$ of duration and viability of the larval phase of Spodoptera frugiperda grown on artificial diet treated with aqueous extracts at $10 \%$ of different vegetative structures of Hedychium coronarium.

\begin{tabular}{lcc}
\hline \multirow{2}{*}{ ESTRUTURAS } & \multicolumn{2}{c}{ FASE LARVAL } \\
\cline { 2 - 3 } & Duração (dias) & Viabilidade (\%) \\
\hline Testemunha & $14,71 \pm 0,17 \mathrm{a}$ & $92 \pm 4,90 \mathrm{a}$ \\
Raiz & $14,72 \pm 0,20 \mathrm{a}$ & $88 \pm 4,90 \mathrm{a}$ \\
Rizoma & $13,88 \pm 0,16 \mathrm{a}$ & $100 \pm 0 \mathrm{a}$ \\
Bainha & $14,08 \pm 0,22 \mathrm{a}$ & $100 \pm 0 \mathrm{a}$ \\
Lâmina Foliar & $13,90 \pm 0,09 \mathrm{a}$ & $96 \pm 4,00 \mathrm{a}$ \\
\hline Valor de $\mathrm{p}$ & $0,0498^{\mathrm{NS}}$ & $0,1153^{\mathrm{NS}}$ \\
\hline CV $(\%)$ & 3,92 & 8,40 \\
\hline
\end{tabular}

*Médias seguidas pela mesma letra na coluna não diferem entre si pelo teste de Tukey $(\mathrm{P} \leq 0,05)$; $\mathrm{NS}=$ não significativo. ${ }^{1} \mathrm{EP}=$ Erro Padrão. ${ }^{*}$ Means followed by the same letter in the column do not differ by Tukey test $(P$ $\leq 0.05) ; N S=$ not significant $.^{l} S E=$ standard error.

Fonte: Autoria própria. Own authorship.

Na pesquisa de Matos et al. (2009), as frações dos frutos de Trichilia catigua A. Juss. (Meliaceae) causaram a morte de $100 \%$ das lagartas da espécie S. frugiperda. Este resultado indica a alta eficiência dessas frações como bioinseticidas, porque ocasionaram a mortalidade nas lagartas testadas logo nas primeiras fases de desenvolvimento, o que poderia gerar a diminuição dos prejuízos na produção agrícola.

Os autores Tanzubil e McCafferry (1990), em seus ensaios realizados com óleo de semente de Azadirachta indica A. Juss. (Meliaceae) sobre S. exempta, concluíram que a 
diminuição do crescimento aliada com a mortalidade, ocasionava nas lagartas um gasto energético maior para que estas pudessem quebrar as substâncias tóxicas que estavam em seu organismo. Assim, ao invés do gasto energético estar direcionado ao crescimento da lagarta e pupação, esta energia estava sendo direcionada para seu sistema de defesa.

As pupas provenientes de lagartas alimentadas com dieta artificial incorporada com extrato aquoso de lâmina foliar de $H$. coronarium apresentaram período de desenvolvimento mais significativamente mais curto. $O$ tratamento com a utilização da raiz também apresentou uma diminuição no período de desenvolvimento quando comparada com a testemunha e os tratamentos com rizoma e bainha (Tabela 4).

$\mathrm{Na}$ fase pupal, o tratamento que mais reduziu a sobrevivência do inseto foi a testemunha, dieta artificial imersa em água destilada, seguida pelo tratamento realizado com o extrato da bainha, quando comparado com os demais tratamentos (Tabela 4). Essa menor viabilidade pupal observada no tratamento testemunha pode estar relacionada a menor massa corporal apresentada pelas lagartas submetidas a este tratamento.

Os pesquisadores Vendramim e Scampini (1997) em seu experimento utilizando o mesmo inseto, sendo alimentados com folhas de milho mergulhadas em extrato aquoso do fruto de Melia azedarach a $10 \%$ (p/v) verificaram ocorrência do alongamento da fase larval, diminuição do peso larval, menor viabilidade larval, além disso, constataram que na fase subsequente de vida do inseto o mesmo apresentou menor peso, duração e a viabilidade pupal.

Tabela 4. Médias ( \pm EP) de duração e viabilidade da fase pupal de Spodoptera frugiperda criada em dieta artificial tratada com extratos aquosos a $10 \%$ de diferentes estruturas vegetativas de Hedychium coronarium. Averages $( \pm S E)$ of duration and viability of pupal phase of Spodoptera frugiperda grown in an artificial diet treated with aqueous extracts at $10 \%$ of different vegetative structures of Hedychium coronarium.

\begin{tabular}{lcc}
\hline \multirow{2}{*}{ ESTRUTURAS } & \multicolumn{2}{c}{ FASE PUPAL } \\
\cline { 2 - 3 } & Duração (dias) & Viabilidade (\%) \\
\hline Testemunha & $11,26 \pm 0,45 \mathrm{a}$ & $56 \pm 12,88 \mathrm{c}$ \\
Raiz & $10,61 \pm 0,25 \mathrm{ab}$ & $96 \pm 4,00 \mathrm{a}$ \\
Rizoma & $11,44 \pm 0,18 \mathrm{a}$ & $100 \pm 0 \mathrm{a}$ \\
Bainha & $11,60 \pm 0,49 \mathrm{a}$ & $60 \pm 9,75 \mathrm{bc}$ \\
Lâmina Foliar & $9,42 \pm 0,30 \mathrm{~b}$ & $83 \pm 4,36 \mathrm{ab}$ \\
\hline Valor de $\mathrm{p}$ & $0,0018^{* *}$ & $0,0001^{* *}$ \\
\hline $\mathrm{CV}(\%)$ & 7,26 & 17,95 \\
\hline
\end{tabular}

** Médias seguidas da mesma letra na coluna não diferem entre si pelo teste de Tukey $(\mathrm{P} \leq 0,05) .{ }^{1} \mathrm{EP}=$ Erro Padrão. ** Means followed by same letter in the column do not differ by Tukey test $(P \leq 0.05)$. ${ }^{l} E P=$ Standard error.

Fonte: Autoria própria. Own authorship.

Rodrigues e Vendramin (1996) citaram que quando o peso pupal das lagartas tratadas com extratos de plantas é menor que o peso pupal do controle, o extrato vegetal diminui a ingestão do alimento pela lagarta, podendo originar adultos pequenos, com possíveis problemas de acasalamento e fêmeas não fecundas, se comparados com indivíduos normais. Outra ação inseticida que poderia ter ocorrido seria causar efeitos deletérios nas lagartas após 
ingestão (COSTA et al., 2004). Lucena (2015), observou que folhas de Piper aduncum L. na concentração de $15,0 \mathrm{mg} \cdot \mathrm{mL}^{-1}$ causaram prolongação e redução do peso pupal de $S$. frugiperda quando comparados a testemunha.

Para a variável massa de adultos não houve diferença significativa entre os tratamentos testados. No entanto, para a variável ciclo total verifica-se que a duração do tratamento com extrato aquoso de lâmina foliar foi estatisticamente menor os tratamentos testemunha, extratos de rizoma e bainha (Tabela 5).

Tabela 5. Médias $( \pm \mathrm{EP})^{1}$ de massa de adultos e duração do ciclo biológico de Spodoptera frugiperda criada em dieta artificial tratadas com extratos aquosos a $10 \%$ de diferentes estruturas vegetativas de Hedychium coronarium. Averages $( \pm S E)^{1}$ of adult mass and duration of the biological cycle of Spodoptera frugiperda grown on artificial diet treated with aqueous extracts at 10\% of different vegetative structures of Hedychium coronarium.

\begin{tabular}{lcc}
\hline ESTRUTURAS & Massa Adulto $(\mathrm{mg})$ & Ciclo Total (dias) \\
\hline Testemunha & $11,72 \pm 4 \mathrm{a}$ & $26,80 \pm 0,58 \mathrm{a}$ \\
Raiz & $12,90 \pm 9 \mathrm{a}$ & $26,13 \pm 0,40 \mathrm{ab}$ \\
Rizoma & $13,78 \pm 6 \mathrm{a}$ & $26,52 \pm 0,46 \mathrm{a}$ \\
Bainha & $12,10 \pm 6 \mathrm{a}$ & $26,66 \pm 0,49 \mathrm{a}$ \\
Lâmina Foliar & $13,14 \pm 7 \mathrm{a}$ & $24,15 \pm 0,38 \mathrm{~b}$ \\
\hline Valor de p & $0,2113^{\mathrm{NS}}$ & $0,0040^{* *}$ \\
\hline CV $(\%)$ & 11,42 & 4,03
\end{tabular}

**Médias seguidas pela mesma letra na coluna não diferem entre si pelo teste de Tukey $(\mathrm{P} \leq 0,05)$; $\mathrm{NS}=$ não significativo. ${ }^{1} \mathrm{EP}=$ Erro Padrão. $* *$ Means followed by the same letter in the column do not differ by Tukey test $(P \leq 0.05) ; N S=$ not significant. ${ }^{l} E P=$ Standard error.

Fonte: Autoria própria. Own authorship.

De modo geral, os extratos aquosos não afetaram negativamente a biologia de $S$. frugiperda. Ao contrário, partes vegetais como a bainha foliar provocaram aumento do peso dos insetos imaturos e redução do tempo de desenvolvimento do inseto.

\section{CONCLUSÃO}

Os extratos aquosos preparados a partir de partes vegetais de Hedychium coronarium não apresentaram toxicidade à Spodoptera frugiperda.

Houve estimulo da alimentação quando se submeteu as lagartas ao extrato aquoso de lâmina foliar, pois as lagartas obtiveram maior massa larval, além de apresentarem um encurtamento da duração da fase pupal.

\section{REFERÊNCIAS BIBLIOGRÁFICAS}

AGUIAR, M. G. A.; PAVARINI, R.; COFFANI-NUNES, J. V.; PAVARINI, G. M. P. Effect of aqueous extract of plant structures of Hedychium coronarium in behavioral action of Sitophilus zeamais. In: INTERNATIONAL SYMPOSIUM OF ENTOMOLOGY, 5, 2015, Viçosa. Proceedings [....] Viçosa: Universidade Federal de Viçosa, 2015. p. 258. 
ALMEIDA, F. A. C.; ALMEIDA, S. A.; SANTOS, N. R.; GOMES, J. P.; ARAÚJO, M. E. R. Efeito de extratos alcoólicos de plantas sobre o caruncho do feijão vigna (Callosobruchus maculatus). Revista Brasileira de Engenharia Agrícola e Ambiental, Campina Grande, v. 9, n. 4, p.585-590, 2005.

BARROS, R. Pragas do milho. In: PEDROSO, R. S. (Coord.). Tecnologia de produção: soja e milho 2011/2012. Maracaju: Fundação MS, 2012. cap. 14, p. 275-296.

BALTAZAR, A. B. S. Uso de açafrão (Curcuma longa L.) para o controle de insetos em milho (Zea mays L.) armazenado. 1994.122 f. Dissertação (Mestrado em Engenharia Agrícola - Pré-Processamento de Produtos Agropecuários) - Faculdade de Engenharia Agrícola, Universidade Estadual de Campinas, Campinas-SP, 1994.

BOEKE, S. J.; BAUMGART, I. R.; VAN LOON, J. J. A.; VAN HUIS, A.; DICKE, M.; KOSSOU. D.K. Toxicity and repellence of African plants traditionally used for the protection of stored cowpea against Callosobruchus maculatus. Journal of Stored Products Research, Doetinchem, v. 40, n. 4, p.423-438, 2004.

BOGORNI, P. C.; VENDRAMIM, J. D. Bioatividade de extratos aquosos de Trichilia ssp. sobre Spodoptera frugiperda (J. E. Smith) (Lepidoptera: Noctuidae) em milho. Neotropical Entomology, Londrina, v. 32, n. 4, p.665-669, 2003.

BRITO, J. P.; OLIVEIRA, J. E. M.; BORTOLI, S. A. Toxicidade de óleos essenciais de Eucalyptus spp. sobre Callosobruchus maculatus (Fabr., 1775) (Coleoptera: Bruchidae). Revista de Biologia e Ciências da Terra, São Cristóvão, v. 6, n. 1, p.96-103, 2006.

COSTA, E. L. N.; SILVA, R. F. P.; FIUZA, L. M. Efeitos, aplicações e limitações de extratos de plantas inseticidas. Acta Biologica Leopoldensia, São Leopoldo, v. 26, n. 2, p.173-185, 2004.

CRUZ, I.; VALICENTE, F. H.; SANTOS, J. P. dos; WAQUIL, J. M.; VIANA, P. A. Manual de identificação de pragas da cultura do milho. EMBRAPA-CNPMS, Sete Lagoas, 1997. $67 \mathrm{p}$.

FARIAS, J. R. Milho Bt e inseticidas no manejo de lepidópteros-praga. 2010. $116 \mathrm{f}$. Dissertação (Mestrado em Agronomia - Produção Vegetal) - Centro de Ciências Rurais, Universidade Federal de Santa Maria, 2010.

FERREIRA, D. F. Sisvar: a computer statistical analysis system. Ciência e Agrotecnologia, Lavras, v. 35, n.6, p.1039-1042, 2011.

GREENE, G. L.; LEPLA, N. C.; DICKERSON, W. A. Velvetbean caterpillar: a rearing procedure and artificial medium. Journal of Economic Entomology, Lanham, v. 69, n. 4, p.487-488, 1976.

ISMAN, M. B. Botanical insecticides, deterrents, and repellents in modern agriculture and an increasingly regulated world. Annual Review of Entomology, Palo Alto, v. 51, n. 1, p.45-66, 2006.

KETOH, G. K.; KOUMAGLO, H. K.; GLITHO, I. A. Inhibition of Callosobruchus maculatus (F.) (Coleoptera: Bruchidae) development with essential oil extracted from 
Cymbopogon schoenanthus L. Spreng. (Poaceae), and the wasp Dinarmus basalis (Rondani) (Hymenoptera: Pteromalidae). Journal of Stored Products Research, Doetinchem, v. 41, n. 4, p.363-371, 2005.

LIMA JUNIOR, I. S.; DEGRANDE, P. E.; MELO, E. P.; BERTONCELLO, T. F.; SUEKANE, R. Infestação de Spodoptera frugiperda (J.E. Smith, 1797) (Lepidoptera: Noctuidae) e seus inimigos naturais em milho nas condições de sequeiro e irrigado. Revista Agrarian, Dourados, v. 5, n. 15, p.14-19, 2012.

LUCENA, D. C. Atividade biológica de extratos de Piper aduncum e Piper hispidinervum sobre Anticarsia gemmatalis e Spodoptera frugiperda. 2015. 66 f. Dissertação (Mestrado em Biotecnologia - Biotecnologia) - Centro de Ciências Biológicas e da Saúde, Universidade de Caxias do Sul, Caxias do Sul, 2015.

MARTINELLI, S.; BARATA, R. M.; ZUCCHI, M. I.; SILVA-FILHO, M. C.; OMOTO, C. Molecular variability Spodoptera frugiperda (Lepidoptera: Noctuidae) populations associated mize and cotton crops in Brasil. Journal of Economic Entomology, Lanham, v. 99, n. 2, p.519-526, 2006.

MATOS, A. P.; NEBO, L.; VIEIRA, P. C.; FERNANDES, J. B.; SILVA, M. F. G. F.; RODRIGUES, R. R. Constituintes químicos e atividade inseticida dos extratos de frutos de Trichilia elegans e T. catigua (Meliaceae). Química Nova, São Paulo, v. 32, n. 6, p.15531556, 2009.

MiCHELOTTO, M. D.; CROSARIOL NETTO, J.; FREITAS, R. S.; DUARTE, A. P.; BUSOLI, A. C. Milho transgênico (BT): efeito sobre pragas alvo e não alvo. Nucleus, Ituverava, Edição Especial, p.67-82, 2013.

MORDUE (LUNTZ), A. J.; NISBET, A. J. Azadirachtin from the neem tree Azadirachta indica: its action against insects. Anais da Sociedade Entomológica do Brasil, Londrina, v. 29, n. 4, p.615-632, 2000.

NAGOSHI, R. N.; SILVIE, P.; MEAGHER, R. L.; LOPEZ, J.; MACHADO, V. Identification and comparison of fall armyworm (Lepidoptera: Noctuidae) host strains in Brazil, Texas, and Florida. Annals of the Entomological Society of America, Cary, N.C., v. 100, n. 3, p.394402, 2007.

PENCOE, N. L.; MARTIN, P. M. Development and reproduction of fall armyworm on several wild grasses. Environmental Entomology, College Park, v. 10, n. 6, p.999-1002, 1981.

ROHDE, C.; MOINO JÚNIOR, A.; SILVA, P. K.; RAMALHO, K. R. O. Efeito de extratos vegetais aquosos sobre a mosca-das-frutas Ceratitis capitata (Wiedemann) (Diptera: Tephritidae). Arquivos do Instituto Biológico, São Paulo, v. 80, n. 4, p.407-415, 2013.

RODRIGUEZ, H. C.; VENDRAMIM, J. D. Toxicidad de extractos acuosos de Meliaceae en Spodoptera frugiperda (Lepidoptera: Noctuidae). Manejo Integrado de Plagas, Turrialba, v. 42, n. 1, p.14-22, 1996.

SÂMIA, R. R.; OLIVEIRA, R. L.; MOSCARDINI, V. F.; CARVALHO, G. A. Effects of aqueous extracts of Copaifera langsdorffii (Fabaceae) on the growth and reproduction of 
Spodoptera frugiperda (J. E. Smith) (Lepidoptera: Noctuidae). Neotropical Entomology, Londrina, v. 45, n. 5, p.580-587, 2016.

SOUSA, A. H.; MARACAJÁ, P. B.; SILVA, R. M. A.; MOURA, A. M. N.; ANDRADE, W. G. Bioactivity of vegetal powders against Callosobruchus maculatus (Coleoptera: Bruchidae) in caupi bean and seed physiological analysis. Revista de Biologia e Ciências da Terra, São Cristóvão, v. 5, n. 2, p.1-5, 2005.

TANZUBIL, P. B.; MCCAFFERY, A. R. Effects of azadirachtin and aqueous neem seed extracts on survival, growth and development of the African armyworm, Spodoptera exempta. Crop Protection, Guildford, v. 9, n. 5, p.383-386, 1990.

VENDRAMIM, J. D.; CASTIGLIONI, E. Aleloquímicos, resistência e plantas inseticidas. In: GUEDES, J. C.; COSTA, I. D.; CASTIGLIONI, E. (Orgs.) Bases e técnicas do manejo de insetos. Santa Maria: Palloti, 2000. cap. 8, p. 113-128.

VENDRAMIM, J. D.; SCAMPINI, P. J. Efeito do extrato aquoso de Melia azedarach sobre o desenvolvimento de Spodoptera frugiperda (J.E. Smith) em dois genótipos de milho. Revista de Agricultura, Piracicaba, v. 72, n. 2, p.159-170, 1997. 